\title{
Simulating Behavior Diversity in BioCrowds
}

\author{
Paulo Knob \\ Department of Computer Science \\ Virtual Humans Laboratory (VHLAB) \\ Pontifical Catholic University of Rio Grande do Sul \\ Porto Alegre, Brazil \\ paulo.knob@edu.pucrs.br
}

\author{
Soraia Raupp Musse \\ Department of Computer Science \\ Virtual Humans Laboratory (VHLAB) \\ Pontifical Catholic University of Rio Grande do Sul \\ Porto Alegre, Brazil \\ soraia.musse@edu.pucrs.br
}

\begin{abstract}
Most of the techniques available nowadays for crowd simulation are focused on a specific situation, e.g. evacuation in hazardous events. Very few of them consider the cultural and personality aspects present in a society to determine the behavior of agents. Therefore, this work aims to build a framework able to deal with different cultural and personality traits as input, and translate them into a group parametrization, which is going to determine the behavior of groups and crowds in virtual environments. Also, we include in BioCrowds a comfort response for agents, in terms of density and thermal characteristics of the environment. Results indicate that the cultural/psychological mappings seem promising, since agents were able to perform as intended. Additionally, agents were able to react due to thermal and density comfort, improving their ability to react to environmental changes.
\end{abstract}

\section{INTRODUCTION}

The area of crowd simulation has been the studying focus for several researchers through many years because of its numerous and varied applications in diverse fields. Although the existence of a large number of techniques in literature [1][5], very few of them take into account the cultural aspects of a nation or society [6]. These aspects can be very useful in providing specific information about a crowd, which can be translated into different behaviors such as desired speed, group cohesion, gesturing, eye gazing, among others. It can be useful to simulate different crowds from all around the world, like an urban simulation running with Chinese agents or a game where the history is set in Germany and, therefore, has Germanic agents.

The goal of this work is to extend the Biocrowds [1] model, making the agent's navigation more realistic using cultural and psychological aspects of people. Therefore, to provide such diversity, it is proposed to use two different methodologies as input to this framework, namely Hofstede's Cultural Dimensions (HCD) [7] and Durupinar [8]. HCD is a very consolidated methodology to evaluate the cultural dimensions of Countries, while Durupinar model states for a computational method to simulate virtual humans based on their personalities. While HCD is not a simulation methodology, we use it as a way to map characteristics in crowds, as will be discussed in this work. Indeed, our framework should be able to receive one of these two cultural/personalities aspects as input, and use them to define group parameters to control virtual agents

\footnotetext{
${ }^{0}$ M.Sc. dissertation
}

in BioCrowds. In addition, we included in BioCrowds the comfort response in terms of density and the thermal characteristics of the space. Our goal is to be able to simulate agents avoiding uncomfortable places in the environment and seek for cozy locations. It is important to notice that cultural, personality aspects and response to comfort relies on more realistic agents reactions w.r.t. environments and groups in the crowd. Our goal is to include various possibilities to simulate behavioral diversity in BioCrowds.

\section{RELATED WORK}

Several ways to simulate crowds were developed in last years. The origin of crowd simulation goes back to Reynolds [9] and Helbing [2] papers. Reynolds [9] simulated flocks of bird-like entities which he called "boids", obtaining a realistic animation using only simple local rules. In its turn, Helbing et al. [2] proposed a psychosocial forces based model to reproduce the pedestrian dynamic. This concept is defined based on the assumption that pedestrians adopt behavioral strategies according stimulus from routine situations. Nowadays, one of the state-of-the-art methods for crowd simulation is ORCA (Optimal Reciprocal Collision Avoidance), proposed by Van der Berg et al. [5]. It is a velocity-based method for collision avoidance between multiple agents, where the main idea is to find the velocity obstacle (VO) between two agents, which defines the zones where a collision should occur between them. Concerning cultural and psychological aspects, the works of Hofstede [7] and Durupinar et al. [8] are worth mentioning. Geert Hofstede proposed the Hofstede's Cultural Dimensions (HCD) [7]. as a framework for cross-cultural communication. It aims to describe the effects of a society's culture based on the values of its individuals, as well how these values can influence on how people behave. For this, six cultural dimensions were defined as percentages values: Power Distance Index (PDI), Masculinity vs. Femininity (MAS), Long-term orientation (LTO), Indulgence vs. restraint (ING), Uncertainty Avoidance (UNC) and Individualism (IND). Durupinar et al. [8] developed a simulation model based on psychological traits which aims to represent emotions and emotion contagion between agents in an effective way. To this end, the OCEAN (Openness, Conscientiousness, Extroversion, Agreeableness, Neuroticism) psychological traits model, proposed by Goldberg [10] is used. 


\section{Proposed Method}

The main proposal of this work is to extend Biocrowds [1] model, by implementing new features and new parameterization, in order to achieve the behavioral diversity aspects, providing more realistic simulation in the virtual world. Figure 1 presents an overview of the method. At the image, it can be seen the three major crowd simulation entities that are going to be tackled in this work: Environment, Groups and Agents.

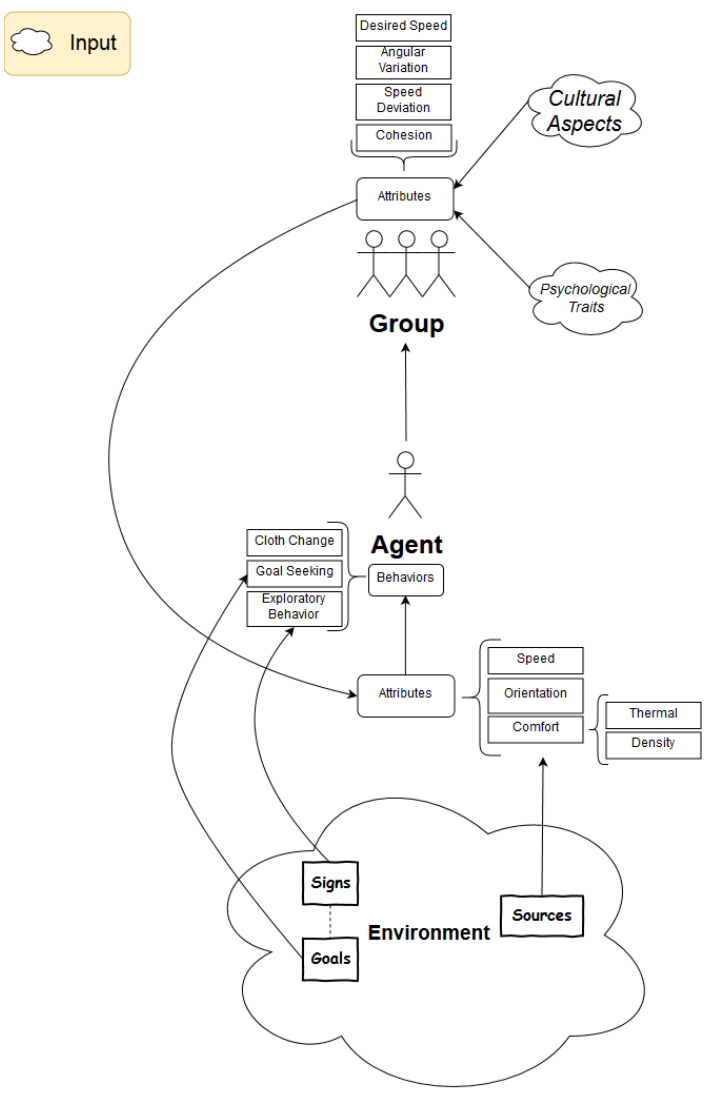

Fig. 1. Overview of the method.

When we think about providing models to endow virtual agents with characteristics that allow the behavioral diversity, we also consider the virtual environment and the way people evolve in such spaces. BioCrowds, as the major part of existent crowd simulators, is a goal based method. It means that virtual agents should "appear" in the simulation knowing their goals and their coordinates in the environment. Having in mind that we wanted to provide diversity of behaviors, we decided to include also an exploratory behavior in the space where agents can be "influenced" by the space and then, even with the same goals, behave in a different way.

Firstly, to achieve the exploratory navigation, we propose to make some changes concerning the goal seeking behavior. In short, instead to have just one goal to achieve, each agent should have a list of desired goals and an intention value for each one of them, representing its willingness to reach them (this factor could be connected with agent personality in a future work). Yet, the exploratory behavior should mimic situations where people do not know where places are located (for example, an agent may be hungry and willing to go to a restaurant, but does not know where it is). Still considering the environment, thermal cells are added in the environment in order to calculate the thermal comfort of the agents, following the proposals of Cheng et al. [11] and Fanger [12].

Regarding the goal to provide behavioral diversity in agents according to cultural and personality aspects, we propose to re-parametrize BioCrowds as follows: i) to define some group parameters (for example, cohesion and desired speed) which will guide agents behaviors. These parameters are defined following mentioned models/theories, like Hofstede cultural dimensions [7]; ii) to consider as input the OCEAN [10] of each agent and compute individual and groups characterization, as well as motion parameters (goals, speed, etc). To do so, we use Durupinar model [8], which work mapped OCEAN factors into agent's behavior.

In our method, a cohesion value $\zeta_{g}$ is set to define how much a group $g$ tends to stay together, in the interval [0,3], where 0 is the lowest cohesion value and 3 is the highest. This interval is defined according to the work of Favaretto et al. [13]. Further, a cohesion distance value $\mu_{g}$ is defined to represent the maximum distance an agent can be away from the rest of the group $g$, without leaving it. This cohesion distance is calculated as follows in Equation 1:

$$
\mu_{g}=H s-\left(\zeta_{g}\left(\frac{H s-H p}{M C}\right)\right),
$$

where $H p$ is the Hall's personal space and $H s$ is the Hall's social space. This distance spaces are described by Hall [14] which defines regions, called by the author "proxemics", that a person tends to maintain to feel comfortable. The $M C$ value stands for Maximum Cohesion and represents the maximum cohesion value a group can achieve (in this work it was used 3 for this value). For instance, if $\zeta_{g}=0$ for a certain group $g$ then $\mu_{g}=3.6$, i.e. this group with low cohesion value can have the members more spread. On the other hand, if $\zeta_{g}=3$ then $\mu_{g}=1.2$, meaning that members stay close to each other in order to be a group, since they have a strong connection and are more attracted.

If an agent gets farther from the rest of the group than the cohesion distance $\mu_{g}$, it is removed from such group and creates a new group for itself. It is defined as follows: $D_{i, g}=$ $d\left(\boldsymbol{p}_{\boldsymbol{i}}, \boldsymbol{p}_{\boldsymbol{g}}\right)$, where $d$ is the Euclidean distance between the agent $i$ position and the center of its group $g$. If it surpasses the value $\mu_{g}$, i.e. $D_{i, g}>\mu_{g}$, this agent leaves the group. In a similar way, if another agent $j$ has its distance to the center of any group $g$ lower or equal than $\mu_{g}$, i.e $D_{j, g}<=\mu_{g}$, and the same immediate goal, it can enter to this group.

Groups of agents have a desired speed to be distributed among the members. Again, we propose to connect this concept with the group parameter. We defined the desired speed initial value of group $g$ as $\psi_{g}=1.2 \mathrm{~m} / \mathrm{s}$. Besides desired speed, it is defined maximum and minimum standard deviation to imply in group members speeds, varying from $\sigma^{\min }=0$ to $\sigma^{\text {max }}=0.2$. So, the individual speed of an agent $A$ is 
determined as a function of group speed $s_{g}$ and a standard variation $\sigma_{g}$ which is computed as follows:

$$
\sigma_{g}=\sigma^{\max }-\left(\left(\sigma^{\max }-\sigma^{\min }\right) \cdot \frac{\zeta_{g}}{\zeta_{\max }}\right),
$$

where $\zeta_{\max }$ stands for higher possible value for cohesion $\left(\zeta_{\max }=3\right)$. Indeed, the speed deviation $\sigma_{g}$ represents a percentage of the desired speed of the group to be randomized for the desired speed of agents of this group. For example, if a group has a desired speed $\psi_{g}=1.0 \mathrm{~m} / \mathrm{s}$ and a speed deviation $\sigma_{g}=0.1$, all agents of this group are going to have a desired speed randomized with $10 \%$ of variation (since $\sigma_{g}=0.1$ ) from the group desired speed. Therefore, all agents would have a desired speed which lies in the interval $[0.9,1.1] \mathrm{m} / \mathrm{s}$, randomly generated.

Besides desired speed and speed deviation, other two parameters are used in order to achieve group behavior: cohesion $\zeta$ and angular variation $\phi$. Just as desired speed, these parameters values can be statically set and are defined, for default, as $\zeta=3$ and $\phi=0$. With all defined, our default groups show high cohesiveness $(\zeta=3)$, no angular variation $(\phi=0)$ and desire to move at a high speed $(s=1.2)$. Since cohesion is maximum, the speed deviation is minimum $(\sigma=0)$;

Now, we can define some group parameters based on cultural and psychological aspects. As discussed by Favaretto et al. [13], we propose that group cohesion $\zeta_{g}$ is a function of $\left(100-M A S_{g}\right)$. Our assumption is that more feminine population can be referred to more cohesive population, as in Equation 3:

$$
\zeta_{g}=\left(\left(\left(100-M A S_{g}\right) \times 3\right) / 100\right),
$$

where $\left(100-M A S_{g}\right)$ gives the feminism percentage aspect of the dimension for group $g$. This value is normalized to lie between 0 and 3, which explains the multiplication by 3 and the posterior division by 100 .

The group desired speed $\psi_{g}$, as described in [13], is calculated as a function of dimension $I N G$. The idea behind is to refer an "indulgent" group as people who are in control of their lives, so it was related to the group speed. If a group is very indulgent (100\% for instance), the group will try to achieve the desired speed. The value of $\psi_{g}$ decreases as the value of $I N G_{g}$, as stated in Equation 4:

$$
\psi_{g}=\left(I N G_{g} \times 1.2 / 100\right)
$$

where the value 1.2 represents the maximum speed that an agent can achieve in the simulator. Therefore, this value must lie between 0 and 1.2.

The angular variation present in the group motion $\phi_{g}$ was related to $(1-L T O)$. Indeed, the assumption is that more angular variation is achieved in groups with lower value of $L T O_{g}$, which states for "Long Term orientation":

$$
\phi_{g}=\left(\left(100-L T O_{g}\right) / 100\right)
$$

where $\phi_{g}$ actually represents a percentage of a maximum angle, which was defined as 90 degrees.

We can also define such parameters as a function of Durupinar's features. The cohesion value $\zeta$ of a certain group $g$ is calculated as presented in Equation 6:

$$
\zeta_{g}=\left(1-\operatorname{Im} p_{g D}\right) \times 3,
$$

where $I m p_{g D}$ represents the Durupinar's impatience (which can be the leader impatience or the average impatience of the group, as explained above). The value is multiplied by 3 in order to keep this parameter between 0 and 3 , as used in this model.

The desired speed value $\psi$ of $g$ is calculated as in Equation 7:

$$
\psi_{g}=1.2 \times\left(\psi_{g D}-1\right),
$$

where $\psi_{g D}$ represents the Durupinar's walking speed (which can be the leader walking speed or the average walking speed of the group, as explained above) and $\left(\psi_{g D}-1\right)$ actually represents a percentage of a defined maximum speed (i.e. 1.2). Since Durupinar's speeds lie between 1 and 2, a simple normalization can give this value.

The angular variation value $\phi$ is described as in Equation 8:

$$
\phi_{g}=1-\left(E e_{g D} / 10\right),
$$

where $E e_{g D}$ represents the Durupinar's exploring environment (which can be the leader exploring environment or the average exploring environment of the group, as explained above) and $\phi_{g}$ actually represents a percentage of a maximum angle, which was defined as 90 degrees. The value is divided by ten in order to normalize $E e_{g D}$.

\section{EXPERIMENTAL RESULTS}

This section aims to present the experimental results achieved by this work and it is divided in three majors parts. Firstly, we show the achieved results in terms of the navigation method developed as an extension to BioCrowds [1], like the state Looking_For (LF) and the intention/signs. Then, we discuss obtained results concerning the cultural and psychological aspects of the simulated crowds, not only for Hofstede, but also for Durupinar approach. Finally, the results achieved with the thermal comfort model are presented.

\section{A. Results obtained with the New Navigation Method}

In order to proceed with the simulations, a 30x20 meters scenario is modeled, with two obstacles (gray shapes) and four goals (in red), illustrated in Figure 2. For each instantiated goal, a sign pointing to it is placed in its exact position. This is done to avoid a non-ending simulation. Otherwise, it would be possible to simulate a scenario with no signs, where agents would never find any goal, since they start the simulation with no knowledge about goal's location. Two signs (in yellow) are placed in the scene: "Sign1" pointing to "Goal1" and "Sign2" pointing to "Goal2". The green selection at the right shows the schedule of the agent, properly ordered by the intention values 
(blue selection). Therefore, the agent starts the simulation in the state LF and wants to achieve Goal3, Goal2, Goal1 and Goal4, respectively.

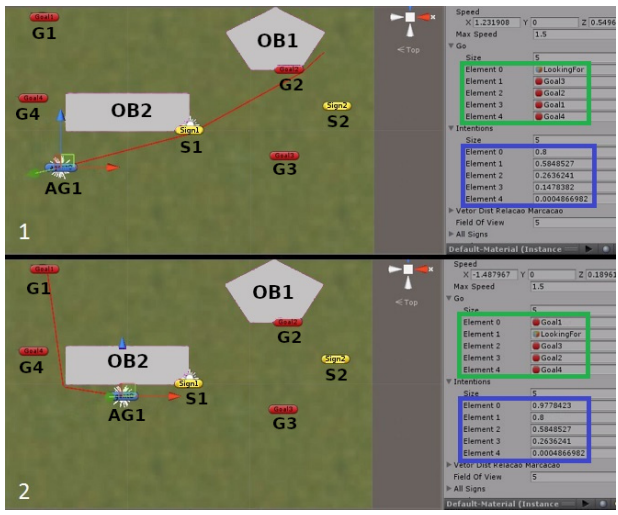

Fig. 2. Two agents following their scheduled goals.

Tests show that interaction with signs works exactly as expected, it means, the two new instantiated signs truly impacts the agent's schedule. The agent starts the simulation looking for a sign, following the schedule which can be seen in Figure 2-1. However, when it perceives "Sign1" (S1), the intention to go to "Goal1" (G1) rises, surpassing the intention to go to "Goal3" (G3) (Figure 2-2). Consequently, the agents status changes from LF to TG, i.e. towards "Goal1" (G1). Also, it is possible to see that the agent starts the simulation with the intention to go to a random location (state Looking_For). Thus, the exploratory behavior is also working as intended.

In order to verify if the quantity of signs truly impact in the simulation time (i.e. the agents arrival time), ten simulations were run with exactly the same parameters, only changing the number of signs. The first simulation had no signs and each subsequent simulation had a new sign added (i.e. 1, 2, ..., 9). A random goal was defined for each sign and its appeal value is set to 1 . It was expected that the more signs, the shorter the simulation time would be. Table I shows simulation mean time and quantity of signs from all ten simulations. It happened in some of the analysis, however, as can be seen from simulations 6 to 10, it seems that this expected behavior is not true. Some hypothesis are raised here. It can be the result of the random process to place new signs and their positions, but it can also show that there is an optimal number of signs in such simulation. Plus, it can just be the effect of the random nature of the state LF. Further analysis are needed in order to properly conclude that.

\section{B. Results Obtained with Cultural Method}

In order to proceed with the subject of cultural simulations, a 30x30 meters scenario is modeled with four goals. The exploratory behavior is deactivated, since it is not necessary here. For all cultural simulations, ten agents are instantiated inside the same group. They have a fixed list of goals to follow, which is Goal2, Goal1, Goal4 and Goal3. Eight test
TABLE I

SIMULATIONS MEAN TIMES WITH QUANTITY OF SIGNS

\begin{tabular}{|c||c||c|}
\hline & Frame Time & Qnt Signs \\
\hline Sim 1 & 8946.8 & 0 \\
Sim 2 & 6471.5 & 1 \\
Sim 3 & 4327.1 & 2 \\
Sim 4 & 4015.7 & 3 \\
Sim 5 & 4040.1 & 4 \\
Sim 6 & 4777.9 & 5 \\
Sim 7 & 2877.7 & 6 \\
Sim 8 & 2458 & 7 \\
Sim 9 & 3014.2 & 8 \\
Sim 10 & 3294.7 & 9 \\
\hline
\end{tabular}

simulations were made, varying the input values. The idea is to check how different input values affect the crowd behavior, both from cultural aspects and psychological traits. Table II shows the achieved results for Hofstede's mapping. After all eight simulations, it is possible to notice that the result expected at the beginning was achieved, it means, groups with higher cohesion values (i.e. Simulations number 1, 2, 5 and 6) presented closest agents and vice-versa. Yet, the desired speed and angular variation seemed to had influence in group behavior, specially to keep the group together, it means, no agents leaving the group.

TABLE II

RESULTS FOR HOFSTEDE's MAPPING

\begin{tabular}{|c||c||c||c||c|c|}
\hline Sim & Time & Max Groups & Avg Speed & Avg Ang & Avg Dist \\
\hline 1 & 126 & 1 & 0,61 & 16,7 & 1,25 \\
\hline 2 & 595 & 1 & 0,13 & 17,77 & 1,19 \\
\hline 3 & 89 & 1 & 0,81 & 11,9 & 2,19 \\
\hline 4 & 628 & 3 & 0,14 & 12,05 & 2,19 \\
\hline 5 & 178 & 2 & 0,55 & 34,8 & 1,32 \\
\hline 6 & 780 & 2 & 0,13 & 38,08 & 1,22 \\
\hline 7 & 122 & 1 & 0,67 & 27,13 & 2,3 \\
\hline 8 & 732 & 1 & 0,12 & 31,13 & 2,2 \\
\hline
\end{tabular}

Figure 3 shows a comparison between the metrics found for both mappings (Hofstede and Durupinar) and the original BioCrowds method, without psychological parameters, illustrated as a constant line. It indicates that both methods, when mapped to BioCrowds, present a similar correspondence. Also, the influence of cultural input on the original behavior of BioCrowds algorithm is clearly observed among all metrics and shown the main goal of this work.

\section{Results Obtained with the Comfort Method}

In order to proceed with the subject comfort simulations, a 22x40 meters scenario is modeled, with eight rooms. Each room has an identifier tag associated, which defines its space functionality. Agents are spawned at the center bottom of the environment, with a random general goal to achieve (i.e. restaurant, shop or theater) which matches one of the possible room's identifier tags. The room's colors denote its actual thermal comfort. Green means it is cozy $\left(t_{a}=18\right)$, blue means it is cold $\left(t_{a}=12\right)$ and yellow means it is hot $\left(t_{a}=26\right)$, where $t_{a}$ represents the air temperature in Celsius. All comfort simulations keep spawning agents until a max defined value is 


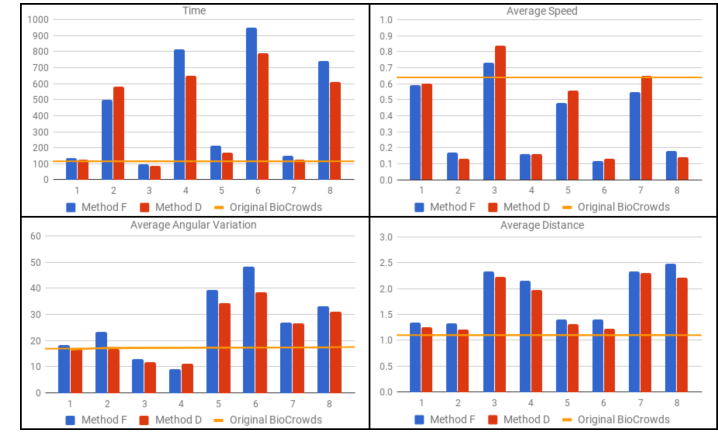

Fig. 3. Comparisons between Method F and Method D. X axis is the simulation identifier (i.e. 1-8) and $\mathrm{Y}$ axis is the value for metrics. On the top/left: time, top/right: the average speed, on the bottom/left: average angular variation and bottom/right: average distance.

achieved (i.e. 100). Agents start the simulation with a random general goal to achieve and medium clothing insulation (i.e. $I_{c l}=1.2$ ). It was ran three test simulations, one with solely thermal comfort, other only with density comfort and a last one with both. The idea is to check if agents avoid uncomfortable places, moving themselves to cozier locations. It is expected that uncomfortable agents search for another place with the same identifier tag, therefore, incommodious rooms should be almost or totally empty.
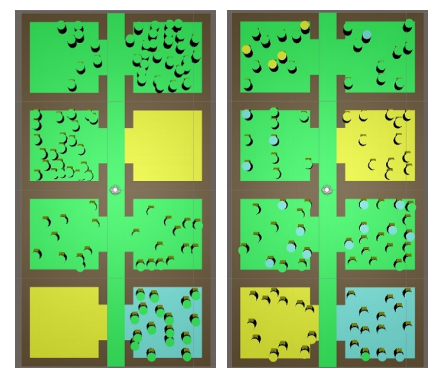

(a) Comfort

Ther

Thermal (b)

fort

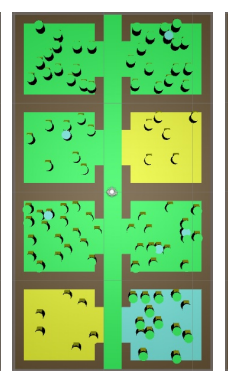

c) Thermal + Density Comforts

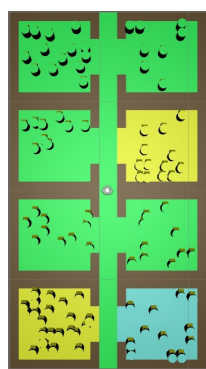

(d) No Comfort
Fig. 4. All three comfort simulation, plus the simulation with no comfort. In (a), no agents were observed inside the hot rooms (yellow rooms). Agents inside the cold room (blue one) are wearing a heavy piece of clothing. In (b), it is possible to notice that agents spread out across all rooms, independent of the temperature. Blue agents are feeling a bit cold and yellow agents are feeling a bit hot. In (c), Blue agents are feeling a bit cold and yellow agents are feeling a bit hot. In (d), simulation with comfort deactivated. Blue agents are feeling a bit cold and yellow agents are feeling a bit hot.

The first simulation was ran using only the thermal comfort. Figure 4(a) shows the final positioning of agents. It is possible to notice that no agents were placed inside the bottom Restaurant, neither inside the first shop from the right (yellow rooms), which were the rooms with an elevated temperature. Some agents were comfortable inside the bottom Theater, which had a low temperature. This can be explained by the clothing insulation of these agents, which were self altered to 2 during the simulation, it means, they are wearing a heavy piece of clothing and feel comfortable inside this room. The second simulation was ran using only the density comfort. Figure 4(b) shows the final positioning of agents. It is possible to notice that agents spread out across all rooms, independent of the temperature. The third simulation was ran using both thermal and density comfort, setting the bias $\alpha=0.5$. Figure 4(c) shows the final positioning of agents. It is possible to notice that, as expected, it seems to be a halfway between only thermal and density methods. The two "hot rooms" (yellow ones) have agents inside, but in a lower number than it can be seen in the density method. At the end, a final simulation was run with the comfort behavior deactivated. The idea was to check if the proposed comfort method is being useful in order to deliver better results than a random choice. Figure 4(d) shows the final positioning of the agents. It can be noticed that agents are just randomly distributed across the eight rooms, according their respective goals.

\section{CONClusion}

This work proposes an extension for Biocrowds model [1] to make its agent's navigation more realistic, while providing agents endowed with characteristics that can generate diversity of behaviors. We propose to re-parametrize BioCrowds based on cultural and psychological dimensions, providing a framework able to simulate cultural crowds. The achieved results seem to be consistent with what was expected. It is interesting to mention about this work validation. All results presented in Section IV show that the framework works as intended, it means, it delivers the expected output, both for the cultural and comfort cases. However, this quantitative evaluation may not be enough to truly answer the question if the crowd is behaving according to its defined cultural parameters or the environment defined thermal/density values. Therefore, a qualitative evaluation could be done in order to complement the results already achieved.

As for future work, there are many things to be done. As already commented, an evaluation with subjects could be conducted with different cultural simulations in order to validate our method. Other cultural and psychological models could be added in our framework (for example, Favaretto dimensions [13]). In fact, following the interactive interface idea implemented in this work, it could be extended to accept any cultural/psychological model. As our framework is, if a new cultural model need to be added, all the formulation need to be hard-coded for it to work properly. One idea is to be able to insert any model, along with its formulation, and the framework would be able to run such cultural/psychological simulations. Finally, the cultural/psychological aspects of the crowd could also be applied to define the willingness of agents to achieve a given goal, as well its susceptibility to react due interaction with signs along the way.

\section{REFERENCES}

[1] A. de Lima Bicho, "Da modelagem de plantas à dinâmica de multidões: um modelo de animação comportamental bio-inspirado," Ph.D. dissertation, Universidade de Campinas, 2009, 114p.

[2] D. Helbing and P. Molnar, "Social force model for pedestrian dynamics," Physical Review E, vol. 51, no. 5, pp. 4282-4286, May 1995.

[3] J. J. Fruin, "Pedestrian planning and design," Metro Asso Urban Dsgners \& Envir Plners, Tech. Rep., 1971, 206p. 
[4] A. Runions, M. Fuhrer, B. Lane, P. Federl, A.-G. Rolland-Lagan, and P. Prusinkiewicz, "Modeling and visualization of leaf venation patterns," ACM Transactions on Graphics, vol. 24, no. 3, pp. 702-711, Jul 2005.

[5] J. Van Den Berg, S. J. Guy, M. Lin, and D. Manocha, "Reciprocal n-body collision avoidance," in Proceedings of the Robotics Research, 2011, pp. 3-19.

[6] D. Lala, S. Thovuttikul, and T. Nishida, "Towards a virtual environment for capturing behavior in cultural crowds," in Proceedings of the 6th International Conference on Digital Information Management. IEEE, 2011, pp. 310-315.

[7] G. H. Hofstede, Culture's consequences: Comparing values, behaviors, institutions and organizations across nations. USA: Sage, 2001, 616p.

[8] F. Durupınar, U. Güdükbay, A. Aman, and N. I. Badler, "Psychological parameters for crowd simulation: From audiences to mobs," IEEE Transactions on Visualization and Computer Graphics, vol. 22, no. 9, pp. 2145-2159, Sep 2016.

[9] C. W. Reynolds, "Flocks, herds and schools: A distributed behavioral model," in Proceedings of the ACM Special Interest Group on Computer Graphics and Interactive Techniques. ACM, 1987, pp. 25-34.

[10] L. R. Goldberg, "An alternative description of personality: the big-five factor structure.” Journal of Personality and Social Psychology, vol. 59, no. 6, pp. 1216-1229, Jun 1990.

[11] L. Chen, C. R. Jung, S. R. Musse, M. Moneimne, C. Wang, R. Fruchter, V. Bazjanac, G. Chen, and N. I. Badler, "Crowd simulation incorporating thermal environments and responsive behaviors," Presence Journal, in press.

[12] P. O. Fanger et al., Thermal comfort. Analysis and applications in environmental engineering. DK: Danish Technical Press, 1970, 244p.

[13] R. M. Favaretto, L. Dihl, R. Barreto, and S. R. Musse, "Using group behaviors to detect hofstede cultural dimensions," in Proceedings of the 2016 IEEE International Conference on Image Processing. IEEE, 2016, pp. 2936-2940.

[14] E. Hall, “The hidden dimension, vol. 1990," USA: Anchor Books, 1969, 240 p.

\section{Publications during the Master Thesis}

[1] L. Dihl, E. S. Testa, P. Knob, G. L. da Silva, R. M. Favaretto, M. F. de Alcantara, and S. R. Musse, Generating cultural characters based on hofstede dimensions, in $\mathbf{2 0 1 7}$ IEEE Virtual Humans and Crowds for Immersive Environments (VHCIE). IEEE, 2017, pp. 1-5.

[2] P. Knob, M. Alcantara, E. Testa, R. Favaretto, G. Lima, L. Dihl, and S. R. Musse, Generating background npcs motion and grouping behavior based on real video sequences, Entertainment Computing, vol. 27, pp. 179-187, 2018.

[3] P. Knob, V. F. de Andrade Araujo, R. M. Favaretto, and S. R. Musse, Visualization of interactions in crowd simulation and video sequences, in 2018 17th Brazilian Symposium on Computer Games and Digital Entertainment (SBGames). IEEE, 2018, pp. 250-259.

[4] P. Knob, M. Balotin, and S. R. Musse, Simulating crowds with ocean personality traits, in Proceedings of the 18th International Conference on Intelligent Virtual Agents. ACM SIGGRAPH Conference, 2018, pp. 233-238.

[5] C. T. Mathew, P. R. Knob, S. R. Musse, and D. G. Aliaga, Urban walkability design using virtual population simulation, in Computer Graphics Forum, vol. 38, no. 1. Wiley Online Library, 2019, pp. 455-469.

[6] R. M. Favaretto, P. Knob, S. R. Musse, F. Vilanova, and A. B. Costa, Detecting personality and emotion traits in crowds from video sequences, Machine Vision and Applications, pp. 1-14, 2018.

[7] V. Araujo, R. M. Favaretto, P. Knob, S. R. Musse, F. Vilanova, and A. B. Costa, How much do you perceive this? an analysis on perceptions of geometric features, personalities and emotions in virtual humans (extended version), arXiv preprint arXiv:1904.11084, 2019.

[8] Andre Da Silva Antonitsch, Diogo Hartmann Muller Schaffer, Gabriel WetzelRockenbach, Paulo Knob, and Soraia Raupp Musse. "BioClouds: A Multi-Level Model to Simulate and Visualize Large Crowds". In: Computer Graphics International (CGI) 2019. Calgary, CA (2019).

[9] Paulo Knob, Gabriel Wetzel Rockenbach, Claudio Rosito Jung and Soraia Raupp Musse. Optimal Group Distribution based on Thermal andPsycho-Social Aspects. In: Computer Animation and Social Agents (CASA) 2019. Paris, FR (2019).

[10] Victor Araujo, Rodolfo Migon Favaretto, Paulo Knob and Soraia Raupp Musse, Felipe Vilanova, Angelo Brandelli Costa. In:Intelligent Virtual Agents (IVA 2019) (Poster). Paris (FR). (Short paper at IVA 2019)

[11] R. M. Favaretto, S. R. Musse, F. Vilanova, and A. B. Costa. (2019). Emotion, Personality and Cultural Aspects in Crowds: Towards a Geometrical Mind. Springer. Contributions in Chapters: Simulating Personality and Cultural Aspects in Crowds; and Generating NPCs Motion Based on Crowd Videos. 